\title{
Measurement and modeling of three-dimensional sound intensity variations due to shallow-water internal waves
}

\author{
Mohsen Badiey ${ }^{a}$ \\ College of Marine Studies, University of Delaware, Newark, Delaware 19716 \\ Boris G. Katsnelson \\ Voronezh State University, Universitetskaya Sq. 1, Voronezh 394006, Russia \\ James F. Lynch \\ Woods Hole Oceanographic Institution, Woods Hole, Massachusetts 02543 \\ Serguey Pereselkov \\ Voronezh State University, Universitetskaya Sq. 1, Voronezh 394006, Russia \\ William L. Siegmann \\ Rensselaer Polytechnic Institute, Troy, New York 12180
}

(Received April 21, 2004; revised October 14, 2004; accepted October 15, 2004)

\begin{abstract}
Broadband acoustic data $(30-160 \mathrm{~Hz})$ from the SWARM'95 experiment are analyzed to investigate acoustic signal variability in the presence of ocean internal waves. Temporal variations in the intensity of the received signals were observed over periods of 10 to $15 \mathrm{~min}$. These fluctuations are synchronous in depth and are dependent upon the water column variability. They can be explained by significant horizontal refraction taking place when the orientation of the acoustic track is nearly parallel to the fronts of the internal waves. Analyses based on the equations of vertical modes and horizontal rays and on a parabolic equation in the horizontal plane are carried out and show interesting frequency-dependent behavior of the intensity. Good agreement is obtained between theoretical calculations and experimental data. (C) 2005 Acoustical Society of America. [DOI: $10.1121 / 1.1828571]$
\end{abstract}

PACS numbers: 43.30.Bp, 43.30.Dr, 43.30.Es, 43.30.Zk [AIT] Pages: 613-625

\section{INTRODUCTION}

The generation of nonlinear internal solitary waves (ISWs) in density-stratified shallow-water regions is typically due to tidal interaction with topographical features. When the bathymetry shoals at the continental shelf, the change in water depth can cause a hydraulic jump condition from which ISW packets can arise and propagate shoreward. Linear internal tides impinging upon the shelf at the socalled "critical angle" can also steepen into nonlinear waves ${ }^{1}$ because of the shoaling bottom. These nonlinear ISWs create a strongly anisotropic (directionally dependent) medium affecting acoustic waves that propagate through them. Sound-speed anisotropy in general can be structural (for example, from sediment layering, where layer formation processes are directional) or dynamical (for example, from a field of internal waves propagating along the continental shelf), but the implication here is that one or more organized processes generate the directionality. Whether the environment is deterministic or random, the anisotropy has the potential of generating directional dependence in acoustic propagation. When broadband acoustic waves are transmitted through an anisotropic medium such as the ISW packets, amplitude and phase fluctuations arise that are strongly related to the characteristics of individual solitons. Quantifying

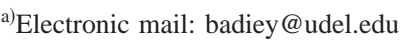

the interaction between these two types of waves is of interest for both underwater acoustics and shallow-water oceanography.

Sound-field fluctuations due to internal waves in shallow-water regions have been studied in several experiments where low-frequency sound propagates in the presence of internal soliton trains. The first paper in this area ${ }^{2}$ examined the frequency spectra variability in the Yellow Sea and recorded significant (up to $20 \mathrm{~dB}$ ) intensity decreases for specific frequencies. This was referred to as an "anomalous attenuation" and was reported as the result of resonant interactions between acoustic waves and the quasiperiodic spatial structure created by an ISW train propagating nearly parallel to the acoustic propagation direction. At nearly the same time another study showed the acoustic field variability in the presence of ISWs. ${ }^{3}$ The acoustic propagation direction was oriented about 10 deg to the ISW fronts, and an adiabatic description of the sound propagation was appropriate for the reported conditions. The intensity showed temporal fluctuations well correlated with water column oscillations from the ISWs, and the amplitude of these fluctuations was about 1-2 dB. In 1995 the multi-institutional SWARM'95 experiment was conducted on the New Jersey continental shelf and showed strong variability, of up to $7 \mathrm{~dB}$, for broadband signal propagation through ISWs. ${ }^{4-7}$ Experimental results presented in 1999 for the Gulf of Mexico ${ }^{8}$ showed relatively small intensity fluctuations that were also correlated with ISWs. In that experiment the angle between the acoustic 


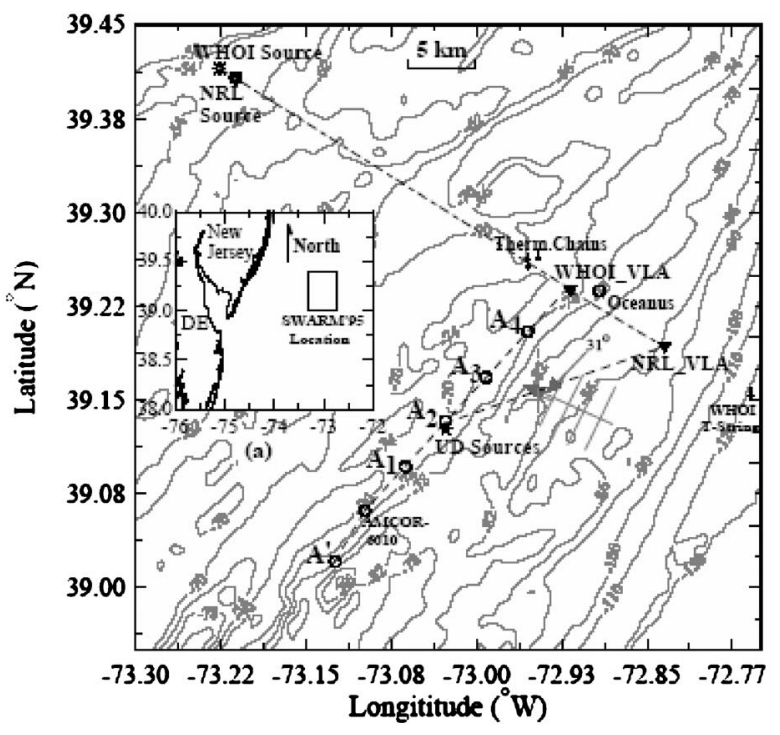

(a)

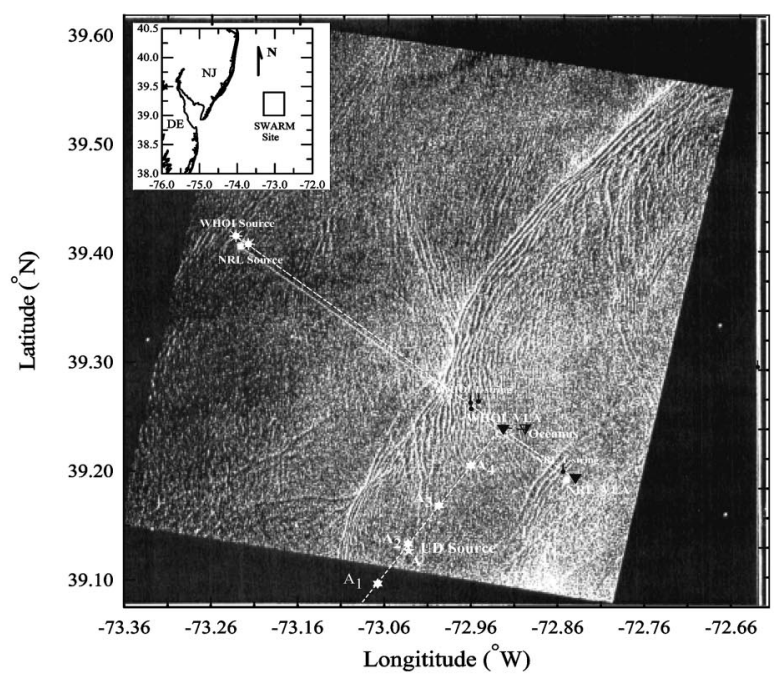

(b)

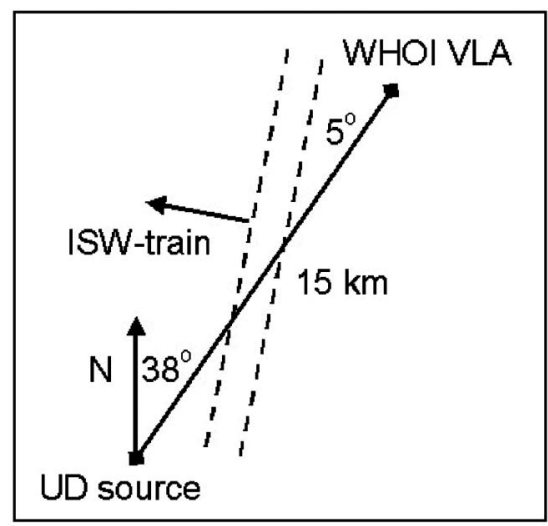

(c)

FIG. 1. (a) Location of SWARM'95 experiment. (b) Satellite picture for 31 August 1995, showing internal wave activity at the experimental site. (c) Schematic diagram of acoustic source and receiver positions relative to the internal solitary wave fronts (dashed lines).

track and ISW fronts was $\beta \sim 30 \mathrm{deg}$, and mode coupling was reported as the reason for the fluctuations. All of the aforementioned work demonstrates two important points: a correlation between behavior of the acoustic signals and the
ISWs, and the significance of the angle between the propagation directions of the acoustic waves and the ISWs. The main feature of these cases that differs from the present work is the orientation of the acoustic track relative to the ISW, which leads to different properties of the acoustic signals.

Along with papers on experiments, a number of theoretical studies appeared over the past few years. Two mechanisms that can cause significant fluctuations of the sound intensity in the presence of ISWs are mode coupling due to ISWs, ${ }^{9,10}$ and "resonant absorption" that can take place if the horizontal spectrum of the ISW train is sufficiently narrow. ${ }^{11,12}$ Another mechanism is horizontal refraction causing focusing and defocusing of intensity in horizontal plane. ${ }^{13,14}$ Modeling of sound fluctuations due to horizontal refraction for conditions of the Sea of Japan showed significant synchronicity of intensity fluctuations in depth for a vertical line array. ${ }^{15}$ Fluctuations of up to $10 \mathrm{~dB}$ in the frequency domain were demonstrated in Ref. 16 to arise from horizontal refraction, depending on the angle $\beta$. Modeling of sound propagation in shallow water in the presence of internal wave fields, both background waves and solitons, was conducted using a fully three-dimensional (3D) PE technique, ${ }^{17,18}$ and significant variations and coherence degradation were observed.

All the above studies have contributed toward the understanding of the complex problem of acoustic signal interactions with ISWs. In this paper we review the experimental observations of intensity fluctuations and provide a theoretical foundation to explain 3D effects on the acoustic field due to the anisotropic nature of the water column resulting from ISWs. The evident cause of the intensity variations is horizontal refraction, a conclusion supported from a different viewpoint by computational results in Ref. 19 .

The paper is organized as follows. In Sec. II the 1995 SWARM experiment is described in detail. Then, a model is presented for a shallow-water channel containing internal waves and with characteristics that generally correspond to the SWARM region. Next, we discuss 3D sound propagation through internal waves, in particular the approach using horizontal rays and vertical modes, the frequency-dependent effects predicted by this approach, and the replacement of the horizontal rays by a parabolic equation. A discussion of results comes next, followed by detailed conclusions and recommendations for future work.

\section{SWARM'95 EXPERIMENT}

The goal of the SWARM'95 experiment was to quantify effects of water column and ocean bottom sound-speed inhomogeneities on acoustic transmissions in shallow regions. ${ }^{1,7}$ The experiment was conducted in the Mid-Atlantic Bight on the continental shelf region directly off Atlantic City, NJ [see Fig. 1(a)], where internal wave activity had been observed via satellite [Fig. 1(b)]. Several research vessels participated in the experiment, including $\mathrm{R} / \mathrm{V}$ OCEANUS, $\mathrm{R} / \mathrm{V}$ CAPE HATTERAS, and R/V ENDEAVOR. Principal investigators in the project included researchers from the Naval Research Laboratory (NRL), Woods Hole Oceanographic Institution (WHOI), the University of Delaware (UD), and the Naval Postgraduate School. Many oceanographic measuring sys- 
tems, as well as two vertical line arrays, were deployed to collect simultaneously both acoustic and detailed environmental data.

In this paper we focus on one part of the experiment, dealing with broadband acoustic signals generated by the UD source and received at the WHOI vertical line array (WVLA) [see Fig. 1(c)]. This array was positioned at $39^{\circ} 14.25^{\prime} \mathrm{N}$ and $72^{\circ} 54.55^{\prime} \mathrm{W}$. It consisted of 16 elements, each with a sampling rate of $1395 \mathrm{~Hz}$, that spanned the water column from 14.9 to $67.4 \mathrm{~m}$ with a spacing of $3.5 \mathrm{~m}$. Five temperature sensors were attached to the WVLA at 12.5, 22.5, 30.5, 50.5, and $60.5 \mathrm{~m}$. This propagation track was designed as UD to WHOI and had the capability to emphasize the azimuthal dependence of the acoustic field from the horizontally anisotropic propagation environment. In particular, the acoustic propagation path ran nearly parallel to the internal wavefronts, in contrast to other paths where different fixed sources were placed so that acoustic tracks ran nearly perpendicular to the internal wavefronts. ${ }^{7}$ Since bathymetry can significantly affect acoustic transmissions, the experimental configuration was chosen to have an essentially flat sea bottom for the entire UD to WHOI track.

During the experiment, numerous sensors were deployed in support of the acoustic measurements. The R/V ENDEAVOR performed high-resolution oceanographic surveys using conductivity-temperature-depth (CTD) casts and tows. High-frequency (200 and $300 \mathrm{kHz}$ ) imaging sonars were used to record internal waves. These measurements were necessary to characterize the generation, propagation, dispersion, and decay of the internal waves and the background field. The environmental data were supplemented by CTD data acquired by the R/V OCEANUS and R/V CAPE HATTERAS and by internally recording oceanographic instrumentation moorings. The latter included two moored ADCP units, six thermistor arrays, and internally recording temperature sensors distributed over the two acoustic receiving arrays. During the experiment, both direct observations and satellite images indicated strong internal wave activity occurring over a large portion of the experiment site. Unfortunately, no satellite images of the internal waves are available during the reported 2-h experimental period.

A 2-h segment of temperature data from 4 August 1995 is shown in Fig. 2. These temperature time series are from thermistor sensors located on the WVLA (12.5 to $60.5 \mathrm{~m}$ from the sea surface). During this period the mixed layer depth is about $15 \mathrm{~m}$, and a negative temperature gradient extends from 15 to $30 \mathrm{~m}$. Figure 3 displays multiple CTD casts that show the envelope of vertical thermocline fluctuations for this day. The sound speed changes from about 1535 $\mathrm{m} / \mathrm{s}$ above the thermocline to $1480 \mathrm{~m} / \mathrm{s}$ below. Both the depth and thickness of the surface and bottom mixed layers change with time. The temperatures in the surface and bottom mixed layers show much less variation than in the thermocline region. The periodicity of the temperature fluctuations shown in the Fig. 2 segment is about 10 to $15 \mathrm{~min}$ and is typical of the entire experimental period. These fluctuations are referred to as short-term, in comparison with the longer-period (about $12 \mathrm{~h}$ ) fluctuations associated with tidal forcing. Although not shown here, similar features are also found in

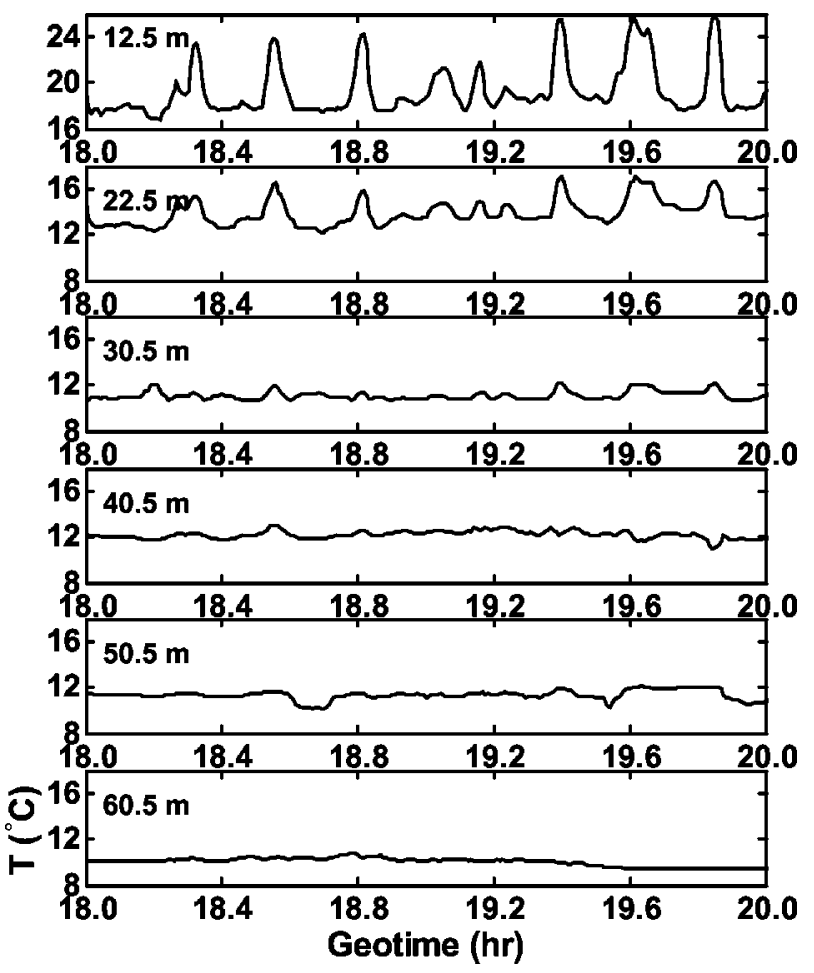

FIG. 2. Measured temperature profiles at six depths on the WHOI VLA versus geotime for 18:00-20:00 GMT on 4 August 1995.

data collected from other temperature sensors at different sites, including the source location.

Throughout the experiment, two different acoustic signals were transmitted from the R/V CAPE HATTERAS every minute. One was from an airgun source (pulse duration $0.1 \mathrm{~s}$ ) and the other was a linear-frequency-modulated (LFM) sweep (pulse duration $30 \mathrm{~s}$ ) transmitted by a J-15 transducer. The source signatures were highly repeatable, as verified by a large number of time series obtained from a monitor hydrophone near the sources. ${ }^{7}$ Although both types of acoustic signals are available for analysis, we focus on the airgun data

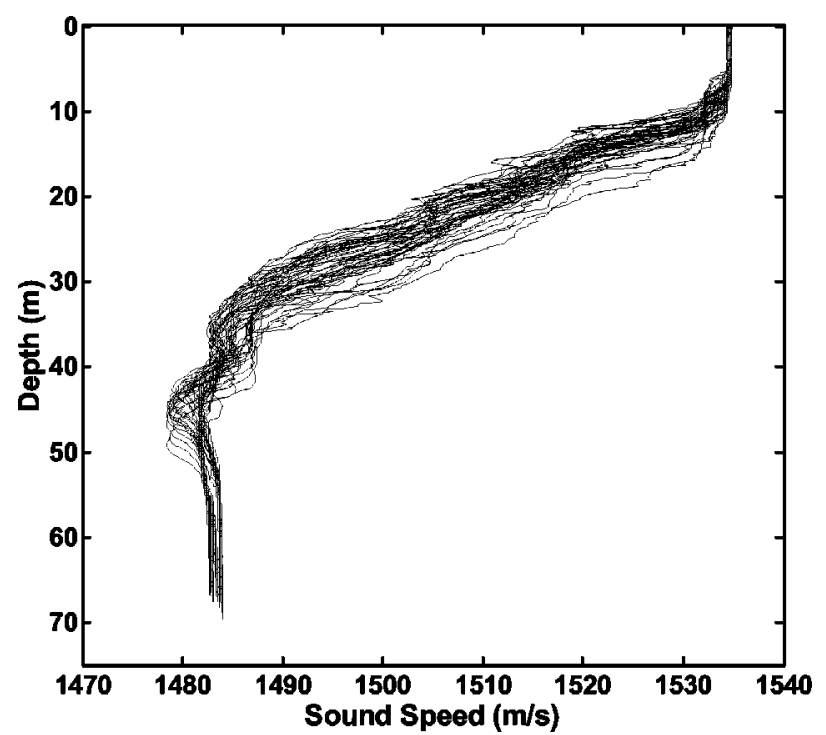

FIG. 3. Sound-speed profiles measured on WVLA showing thermocline fluctuations during the passage of ISWs for 18:00-20:00 GMT on 4 August 1995. 

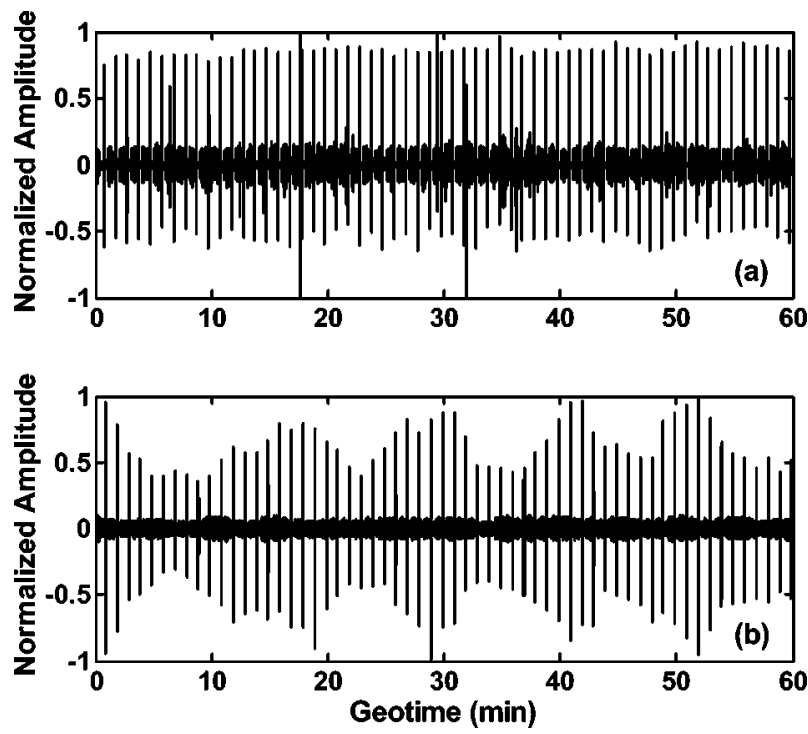

FIG. 4. Normalized pressure amplitude versus geotime from 19:00 to 20:00 GMT on 4 August 1995, measured: (a) at $2 \mathrm{~m}$ from the source, and (b) on the WHOI VLA $14.98 \mathrm{~km}$ from the source at a hydrophone $45 \mathrm{~m}$ from the sea surface. Note that amplitude variations at the source are negligible compared to those at the receiver.

here. The frequency spectrum of the monitor phone, also shown in Ref. 7, is dominated by peak at $32 \mathrm{~Hz}$ (with halfpower width about $5 \mathrm{~Hz}$ ), plus much smaller peaks at harmonics of this fundamental.

While the source ship stayed at a fixed location for several hours, the airgun source transmitted acoustic signals approximately every minute. During the interval 18:00 to 20:00 GMT on 4 August 1995, the source was placed at $12 \mathrm{~m}$ below the sea surface (just above the thermocline) at position A2 in Fig. 1(a), about $15 \mathrm{~km}$ from the WVLA. A segment of acoustic data from geotime 19:00 to 20:00 GMT is shown in Fig. 4. The reference hydrophone collected the data at the source location, and the received signals are from the WVLA. Signals were transmitted every 60.038 s. The nearsource airgun signatures [Fig. 4(a)] show nearly constant amplitude over time, whereas the corresponding WVLA signals show considerable fluctuations over the hour [Fig. 4(b)]. The approximately periodic fluctuations of amplitude observed in the arrivals (the spikes) are due to internal waves that were present between the source and receiver during this period, as will be demonstrated in subsequent sections.

The WVLA received signals show strong fluctuations in amplitude versus geotime, indicating acoustic scattering by a fully developed ISW packet along the source-receiver path. Comparison of Figs. 2 and 4 shows correlation between the patterns of the internal waves and the acoustic signals. In particular, the acoustic and oceanographic fluctuations have essentially the same periodicity.

The source spectrum $S(\omega)$ of the radiated signal $f(t)$ is

$$
S(\omega)=\frac{1}{2 \pi} \int_{-\infty}^{\infty} f(t) e^{i \omega t} d t,
$$

where $f(t)$ represents the pressure signature of the source. The total radiated pulse energy is

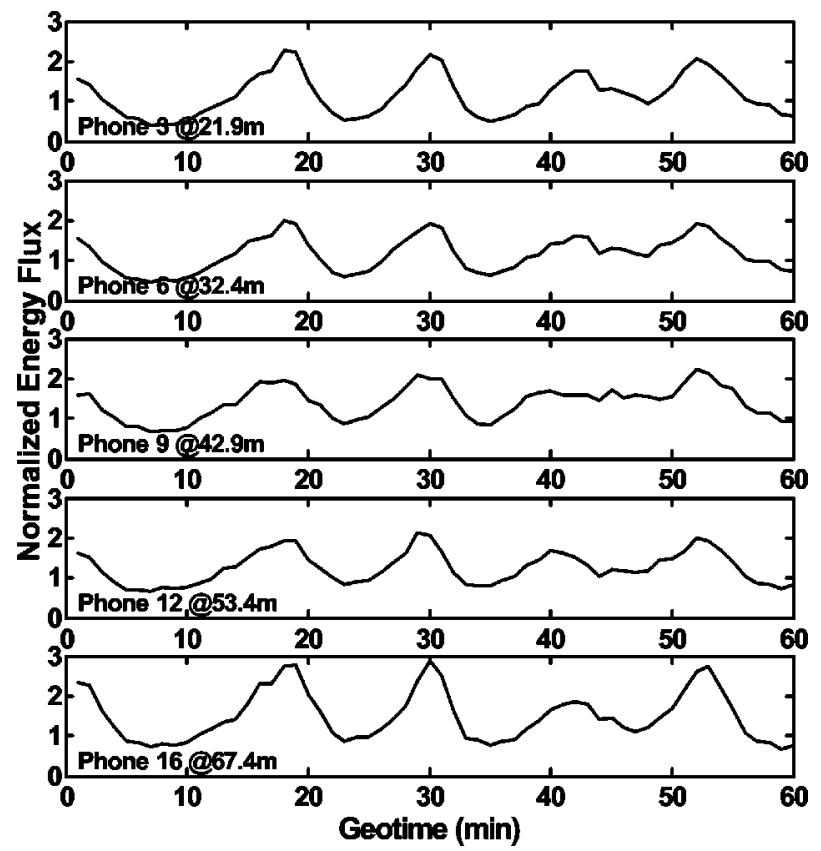

FIG. 5. Total time-integrated intensity $I(z, T)$ versus geotime for signals at five receiver depths.

$$
E_{0}=\frac{1}{4 \pi \rho c} \int_{-\infty}^{\infty} f^{2}(t) d t=\frac{1}{\rho c} \int_{0}^{\infty}|S(\omega)|^{2} d \omega
$$

Using the estimated airgun source power with a pulse duration of $\sim 0.1 \mathrm{~s}$, combined with the amplitude of the sound field at a distance of $2 \mathrm{~m}(\sim 15 \mathrm{kPa})$, the source level at $1 \mathrm{~m}$ is calculated to be $210 \mathrm{~dB}$ re: $1 \mu \mathrm{Pa}$. In this paper we use the spectrum of the source monitor ${ }^{7}$ for $S(\omega)$ and calculate all frequency integrals over 30 to $160 \mathrm{~Hz}$. Because of our objectives here, it is not necessary to account for differences between the measured spectra of the monitor and the actual spectra of the source. ${ }^{12}$

For subsequent derivations we denote the sound pressure of the received signal by $p\left(z_{j}, t, T_{i}\right)$, where $T_{i}$ is the geotime (in 1-min intervals, denoted by the subscript) from 19:00 to 20:00 GMT and $z_{j}$ is the $j$ th hydrophone on the WVLA. The indices $i$ and $j$ are omitted where no confusion occurs.

The total time-integrated intensity (i.e., Energy Flux) of a received acoustic pulse at depth $z$ on the WVLA is represented by

$$
I(z, T)=\int_{T}^{T+\Delta t} \frac{p^{2}}{\rho c} d t,
$$

where $\Delta t$ is the duration of received pulse $(\sim 1 \mathrm{~s})$. Equation (3) was calculated for each of the 60 pulse signals received during that hour, and the results are shown for five representative WVLA elements in Fig. 5.

Several features of the fluctuations in Fig. 5 deserve detailed discussion. First, significant amplitude fluctuations occur, of up to $7 \mathrm{~dB}$. This value cannot be explained simply by local displacements of the thermocline level. Estimates of the intensity fluctuations due to changes of the sound-speed profile via thermocline displacements along a 2D slice (the usual acoustic path) produce only up to $15 \%$ variation in the received intensity. Thus, some explanation beyond a $2 \mathrm{D}$ physi- 


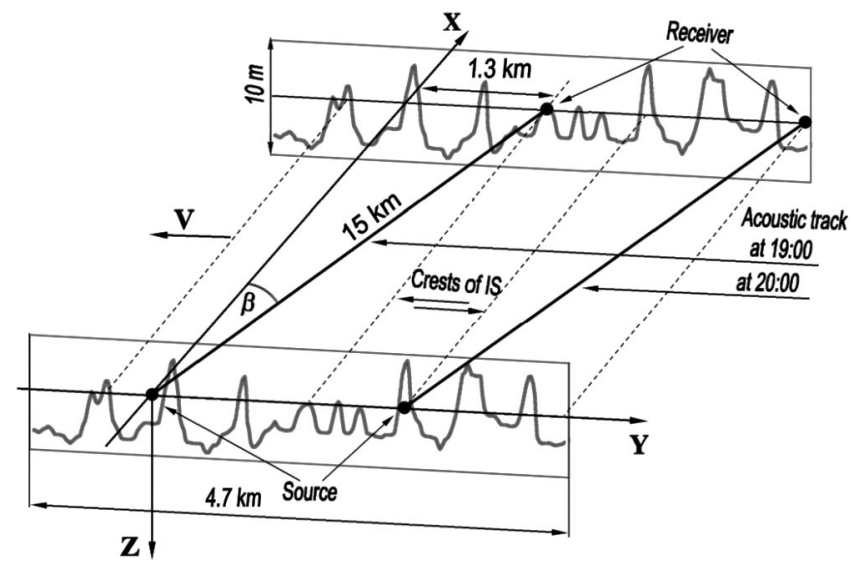

FIG. 6. Schematic diagram of the source-receiver track useful for modeling. The region between the two solid parallel lines corresponds to the geotime period between 19:00 and 20:00 on 4 August 1995 .

cal mechanism is needed. Second, amplitude fluctuations at different depths show only weak dependence on depth. Moreover, they are effectively synchronous, meaning that at any geotime a simultaneous increase or decrease takes place for practically all depths, and show small increases near the bottom. Thus, they cannot result from changes in modal interference patterns, which generally stem from thermocline oscillations near the receiver. Third, the received acoustic signals correlate closely with ISW oscillations. The number and period of the oscillations in the thermocline variability from 19:00 to 20:00 GMT (Fig. 2) correspond to the same quantities observed in the fluctuations of energy flux (Fig. 5).

The first two observations above cannot be explained by interference between waveguide modes. However, they can quite naturally be interpreted as manifestations of 3D horizontal refraction.

\section{SHALLOW-WATER CHANNEL MODEL WITH INTERNAL WAVES}

To model the shallow-water waveguide in the experiment, a Cartesian coordinate system is chosen with $x$ and $y$ in the horizontal plane and $z$ axis directed downwards, as shown in Fig. 6. The air-sea interface corresponds to $z=0$ and the flat bottom is $z=H=70 \mathrm{~m}$. The sound source is at the point $\mathbf{r}_{S}=0, z_{S}$, and the receiving array is at $r_{R}$ $=\left(x_{R}, y_{R}\right)$ with a source-receiver separation $L \sim 15 \mathrm{~km}$. Along the track the sound-speed profile is $c(\mathbf{r}, z, T)=c_{0}(z)$ $+\delta c(\mathbf{r}, z, T)$, where $c_{0}(z)$ is the mean sound-speed profile in the absence of ISWs and $\delta c(\mathbf{r}, z, T)$ is the perturbation due to internal waves. The vector $\mathbf{r}=(x, y)$ denotes a point in the horizontal plane. The mean profile, obtained from the experimental data ${ }^{7}$ in Fig. 3, consists of a thermocline layer extending about $25 \mathrm{~m}$ between two near-isospeed layers. The sound-speed variation between the isospeed layers is over 50 $\mathrm{m} / \mathrm{s}$, which gives a large value $\left(\sim 2 \mathrm{~s}^{-1}\right)$ for the vertical gradient of the thermocline. The geoacoustic parameters in the bottom are selected as sound speed $c_{1}=1750 \mathrm{~m} / \mathrm{s}$, $\rho_{1}=1.8 \mathrm{~kg} / \mathrm{m}^{3}$, and attenuation coefficient $\alpha_{f}=0.25 \mathrm{~dB}$ $/(\mathrm{km} \cdot \mathrm{Hz})$, based on previous work at the SWARM site. ${ }^{1}$

Next, a time-varying model is needed for sound-speed fluctuations in the water column for any geotime $T$ and lo- cation. For this purpose isopycnal (that is, isodensity) surfaces $\zeta(\mathbf{r}, z, T)$ are constructed from the experimental data. This approach is used, rather than an analytical model, in order to preserve more of the detail in the measured soundchannel fluctuations. The ISW packet is assumed to have a plane wavefront parallel to the $x$ axis and to move at speed $V$ in the negative- $y$ direction

$$
\zeta(\mathbf{r}, z, T)=\zeta\left(\mathbf{r}_{R}, z, T+\frac{y-y_{R}}{V}\right) .
$$

The parameter $V$ was not measured directly from the ISW wavefronts but rather is estimated from KdV soliton theory. The Appendix provides an approximate value for $V$ $\sim 0.65 \mathrm{~m} / \mathrm{s}$ at the SWARM site, which is in reasonable agreement with other estimates of this parameter made there. Note that the acoustic track has an angle of $\beta \sim 5$ deg with the $x$ axis, so the source and receiver are on different ISW fronts for a given geotime (see Fig. 6). The spatial shift along the $y$ axis between the source and receiver is $y_{R} \sim 1300 \mathrm{~m}$.

Equation (4) leads to a perturbation of the sound-speed profile due to the ISW

$$
\delta c(\mathbf{r}, z, T)=Q c_{0}(z) N^{2}(z) \zeta(\mathbf{r}, z, T),
$$

where $N(z)=(g d \rho / \rho d z)^{1 / 2}$ is the Brunt-Vaisala frequency and $Q \sim 2.4 \mathrm{~s}^{2} / \mathrm{m}^{20}$ To obtain $\zeta$ isothermal, rather than isopycnal, displacements in the data are tracked, since vertical profiles of both salinity and temperature are required for isopycnals. This approximation introduces only a small error. In shallow water the main part of the energy of an ISW is in its first mode $\Phi(z)$, so that the displacement in Eq. (5) is written as $\zeta(\mathbf{r}, z, T)=\Phi(z) \zeta_{s}\left[\mathbf{r}_{R}, T+\left(y-y_{R}\right) / V\right]$. The modal normalization condition is $\max (\Phi)=1$, so $\zeta_{s}$ is the modeled displacement of the isopycnal at the depth where $\Phi(z)=1$.

\section{3D SOUND PROPAGATION THROUGH INTERNAL WAVES}

The complex amplitude of the sound field due to a broadband source is expressed by the spectral Fourier integral

$$
P(\mathbf{r}, z, t)=2 \int_{0}^{\infty} S(\omega) \Psi(\mathbf{r}, z, \omega) e^{-i \omega t} d \omega,
$$

where $S(\omega)$ is the spectrum of the radiated signal and $\Psi(\mathbf{r}, z ; \omega)$ is a spectral component of the sound-pressure field at any point $(\mathbf{r}, z)$. For brevity the geotime parameter $T$ and source coordinates $\left(\mathbf{r}_{s}, z_{s}\right)$ are omitted from the arguments of $\Psi$ (and elsewhere where no confusion arises). The pressure component satisfies the inhomogeneous Helmholtz equation with a point source of unit amplitude on its right side. A series expansion of this Green's function $\Psi$ in modal eigenfunctions yields

$$
\Psi(\mathbf{r}, z, \omega)=\sum_{l} \psi_{l}(\mathbf{r}, z, T) P_{l}(\mathbf{r}),
$$

where the $\psi_{l}(\mathbf{r}, z, T)$ are the adiabatic (local) vertical modes and the $P_{l}$ are the modal amplitudes. The complex eigenval- 

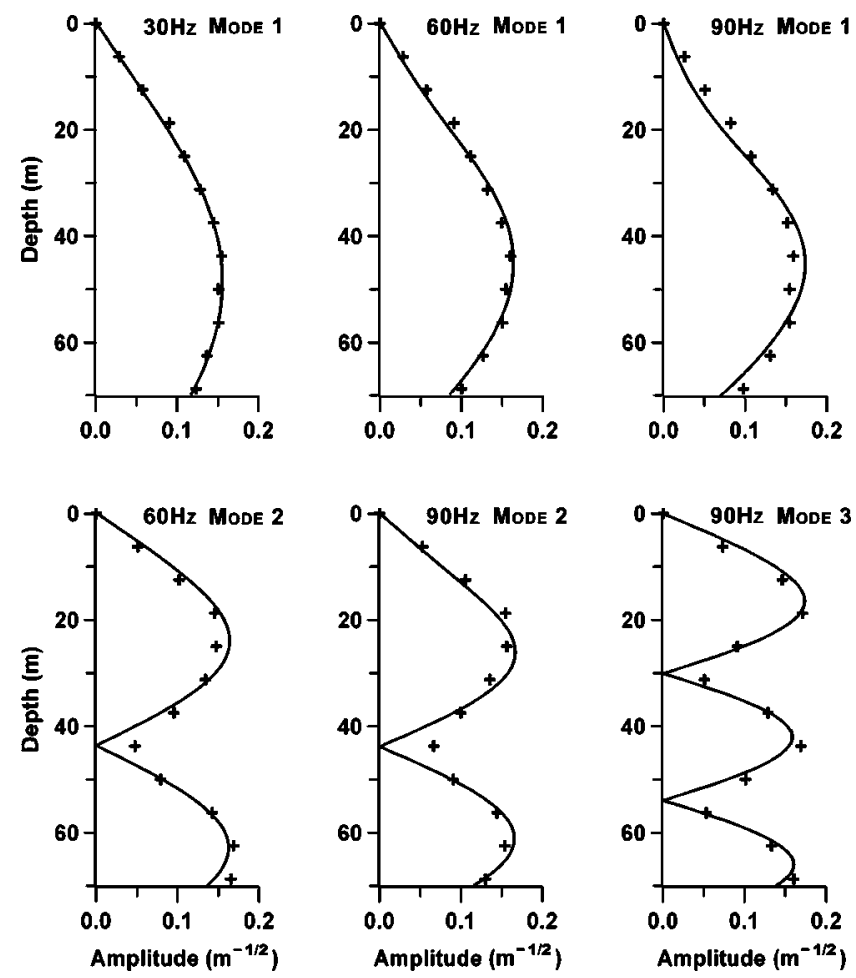

FIG. 7. Normalized vertical mode functions (modes 1 and either 2 or 3 ) for frequencies 30, 60, and $90 \mathrm{~Hz}$. Solid curves show theoretical results based on Eq. (9); crosses show modal decomposition results from data.

ues are expressed as $\xi_{l}=q_{l}+\gamma_{l} / 2$, and can be obtained from the boundary value problem

$$
\begin{aligned}
& \frac{d^{2} \psi_{l}(\mathbf{r}, z, T)}{d z^{2}}+\left\{\frac{\omega^{2}}{\left[c_{0}(z)+\delta c(\mathbf{r}, z, T)\right]^{2}}-\xi_{l}^{2}(\mathbf{r}, T)\right\} \\
& \times \psi_{l}(\mathbf{r}, z, T)=0,
\end{aligned}
$$

subject to the usual surface and bottom boundary conditions and interface conditions. Normalized vertical modes calculated from Eq. (8) for different frequencies at the receiver $\mathbf{r}$ $=\mathbf{r}_{R}$ and geotime 19:00 are shown in Fig. 7, along with modes extracted from the SWARM data. Excellent agreement between the calculated and the experimental modes indicates that the parameters selected for the sound-speed formulas adequately represent the experimental waveguide.

Next, Eq. (8) is substituted into Eq. (7) and mode coupling terms are neglected, which is consistent with the adiabatic mode assumption. ${ }^{21}$ The latter is a reasonable approximation for along-crest propagation in the modeled waveguide. Therefore, the modal amplitudes $P_{l}(\mathbf{r})$ satisfy the equation

$$
\left[\frac{\partial^{2}}{\partial x^{2}}+\frac{\partial^{2}}{\partial y^{2}}+\xi_{l}^{2}(\mathbf{r})\right] P_{l}(\mathbf{r})=0 .
$$

Equation (9) is a 2D Helmholtz equation in which the squared adiabatic eigenvalues play the role of the squared wave vector (which itself is proportional to the refraction index). In the present case $\xi_{l}$ is complex and its imaginary part incorporates modal attenuation.

The total time-integrated intensity of a broadband signal at any point is expressed from Eqs. (3), (6), and (7) by

$$
I(\mathbf{r}, z)=\frac{4 \pi}{\rho c} \int_{0}^{\infty} d \omega|S(\omega)|^{2} \sum_{l}\left|P_{l}(\mathbf{r})\right|^{2} \psi_{l}^{2}(\mathbf{r}, z) .
$$

As usual in such calculations, the cross terms that appear in Eq. (10) (those with $l \neq m$, where $m$ is the other summation index) are neglected. The justification relies on the spatial scale of the cross terms being determined by $\Lambda_{l m}$, which is the interference wavelength that is inversely proportional to the difference of horizontal wave numbers $l$ and $m$. Specifically, integration over frequency from Eq. (6) corresponds to a summation of terms from Eq. (7) with different frequencydependent scales $\Lambda_{l m}$ of interference beating. If the signal frequency bandwidth $\delta \omega \geqslant \omega \Lambda_{l m} / L$, then the cross terms are negligible. For our situation $\delta \omega \sim 130 \mathrm{~Hz}, \Lambda_{l m} \leqslant 1 \mathrm{~km}$, the center frequency $\omega$ is no larger than $150 \mathrm{~Hz}$, and $L$ $\sim 15 \mathrm{~km}$, so the condition for elimination of the cross terms is easily satisfied. The main quantity to be calculated is $P_{l}(\mathbf{r})$ from Eq. (9).

\section{A. Horizontal rays and vertical modes}

A 2D ray solution for Eq. (9) has been published by Weinberg and Burridge using their theory of "horizontal rays (HRs) and vertical modes". 22 The function $P_{l}(\mathbf{r})$ is assumed in the form

$$
P_{l}(\mathbf{r})=\sum_{m} A_{m l}(\mathbf{r}) \exp \left[i \theta_{m l}(\mathbf{r})\right],
$$

where $A_{m l}(x, y)$ is the amplitude and $\theta_{m l}(x, y)$ is the eikonal for the $m$ th HR component of the $l$ th vertical mode $\psi_{l}(\mathbf{r}, z)$. In general, multiple HRs for a given vertical mode can reach the point of observation. These rays have separate trajectories and different amplitudes and eikonals. Thus, a summation over all horizontal eigenrays (i.e., with the index $m$ ) is required. The amplitude and the eikonal satisfy traditional $2 \mathrm{D}$ equations of ray acoustics ${ }^{21,23}$

$$
\begin{aligned}
& \left(\nabla_{r} \theta_{m l}\right)^{2}=q_{l}^{2}(\mathbf{r}), \\
& 2 \nabla_{r} A_{m l} \nabla_{r} \theta_{m l}+A_{m l} \nabla_{r}^{2} \theta_{m l}+q_{l} \gamma_{l} A_{m l}=0,
\end{aligned}
$$

where $\nabla_{r}=(\partial / \partial x, \partial / \partial y)$.

Ray trajectories in the horizontal plane are found from the ordinary differential equations

$$
\begin{aligned}
& \frac{d \mathbf{r}_{m l}}{d t}=U_{l} \boldsymbol{\tau}_{m l}, \\
& \frac{d \mathbf{q}_{m l}}{d t}=U_{l} \nabla q_{l},
\end{aligned}
$$

where $d t=U_{l}^{-1} \sqrt{(d x)^{2}+(d y)^{2}}$ is a measure of arrival time for mode $l, \boldsymbol{\tau}_{m l}(\mathbf{r})=\nabla_{\mathbf{r}} \theta_{m l}(\mathbf{r}) /\left|\theta_{m l}(\mathbf{r})\right|$ is a unit tangent vector to the horizontal ray, $\nabla_{\perp}=\nabla_{\mathbf{r}}-\boldsymbol{\tau}\left(\boldsymbol{\tau} \nabla_{\mathbf{r}}\right)$ is the transverse gradient, $U_{l}=\left(d q_{l} / d \omega\right)^{-1}$ is the group velocity of the $l$ th vertical mode, and $\mathbf{q}_{n l}=q_{l} \boldsymbol{\tau}_{n l}$ is the wave vector (tangent to ray $n$ for vertical mode $l$ ). Equations (14) and (15) describe space-time HRs, ${ }^{23}$ because arrival time is the ray parameter. Using these equations one can find both the trajectories of the HRs and arrival times of the corresponding modal pulses. These equations must be accompanied by conditions at the source $\mathbf{r}_{s}$ such that $\left.\mathbf{r}_{l}\left(t ; \mathbf{r}_{S}, \boldsymbol{\tau}_{S}\right)\right|_{t=0}=\mathbf{r}_{S}$ and 
$\left.\mathbf{q}_{l}\left(\mathbf{r}_{l}, t ; \mathbf{r}_{s}, \boldsymbol{\tau}_{s}\right)\right|_{\mathbf{r}_{l}=\mathbf{r}_{s}, t=0}=q_{l}\left(x_{s}, y_{s}\right) \boldsymbol{\tau}_{s}$. If one defines $\boldsymbol{\tau}_{S}$ $=\left(\cos \chi_{S}, \sin \chi_{S}\right)$, where $\chi_{S}$ is the HR angle at the source, then $\chi_{S}$ and $t$ can be considered as ray coordinates. Also, if $\chi_{S}$ is fixed, a separate HR can be obtained, and for fixed $t$, a wavefront exists in the horizontal plane. The HRs allow construction of the eikonal function along a ray path

$$
\theta_{l}(\mathbf{r}, t)=\theta_{l}\left(\mathbf{r}_{s}, 0\right)+\int_{0}^{t} U_{l} q_{l}\left[\mathbf{r}\left(t^{\prime}\right)\right] d t^{\prime}
$$

Because the spatial scale of $q_{l}$ in the horizontal plane is $\left|q_{l} / \nabla_{r} q_{l}\right|$, this value must be much greater than a wavelength for applicability of HR theory. The amplitude of the HRs can be written as

$$
A_{l}(t)=A_{l}(0) / \sqrt{J} \exp \left\{i \theta_{l}(\mathbf{r}, t)-\int_{0}^{t} \gamma_{l}\left[\mathbf{r}\left(t^{\prime}\right)\right] / 2 d t^{\prime}\right\},
$$

where $J(t)$ describes the divergence of HRs; $J(t)$ $=d \Omega_{t} / d \Omega_{0}$ is the ratio of the lengths of corresponding pieces of wavefronts along a ray tube.

An effective squared wave vector in the horizontal plane is on the right side of Eq. (12). Equivalently, an effective mode-dependent refraction index can be introduced, $n_{l}(\mathbf{r})$ $=q_{l}(\mathbf{r}) / q_{l}^{0}$, where $q_{l}^{0}$ is an eigenvalue for the waveguide without ISWs. Because of the small perturbation $|\delta c| \ll c_{0}$, which correspondingly implies $\left|q_{l}-q_{l}^{0}\right| \ll q_{l}^{0}$, the refraction index for HRs is a sum of the unperturbed value 1 and a small perturbation $\mu_{l}$ due to ISWs, which depends upon horizontal coordinates and $T$

$$
n_{l}^{2}(\mathbf{r}, T)=1+\mu_{l}(\mathbf{r}, T)
$$

The quantity $\mu_{l}$ has appeared in various publications (for example, see Ref. 15)

$$
\begin{aligned}
\mu_{l}(\mathbf{r}, T) & =-\frac{2 Q k^{2}}{\left(q_{l}^{0}\right)^{2}} \int_{0}^{H}\left[\psi_{l}^{0}(z)\right]^{2} N^{2}(z) \Phi(z) \zeta(\mathbf{r}, z, T) d z \\
& =-\nu_{l} \zeta_{s}\left(\mathbf{r}_{R}, T+\frac{y-y_{R}}{V}\right)
\end{aligned}
$$

The second equality in Eq. (19) uses the two factors $\nu_{l}$ and $\zeta_{s}(\mathbf{r}, T)$, where $\zeta_{s}$ was explained in Sec. III and is determined by the shape of the ISW train. The other factor

$$
\nu_{l}=\frac{2 Q k^{2}}{\left(q_{l}^{0}\right)^{2}} \int_{0}^{H}\left[\psi_{l}^{0}(z)\right]^{2} N^{2}(z) \Phi(z) d z
$$

depends on the waveguide parameters.

The refractive index in the horizontal plane depends on frequency through its dependence on the eigenfunction, because of the overlap of the eigenfunction with the thermocline layer. ${ }^{16}$ Generally the eigenfunctions are not strongly frequency dependent. However, the portion of the eigenfunction overlapping the thermocline can be sensitive to frequency (see, for example, Fig. 7). Values of $\left|\bar{\mu}_{l}\right|=\nu_{l} \zeta_{s}$ are shown in Fig. 8 as a function of frequency and characterize the refractive index when the ISW amplitude $\zeta_{s}$ is at its maximum $(\sim 10 \mathrm{~m})$.

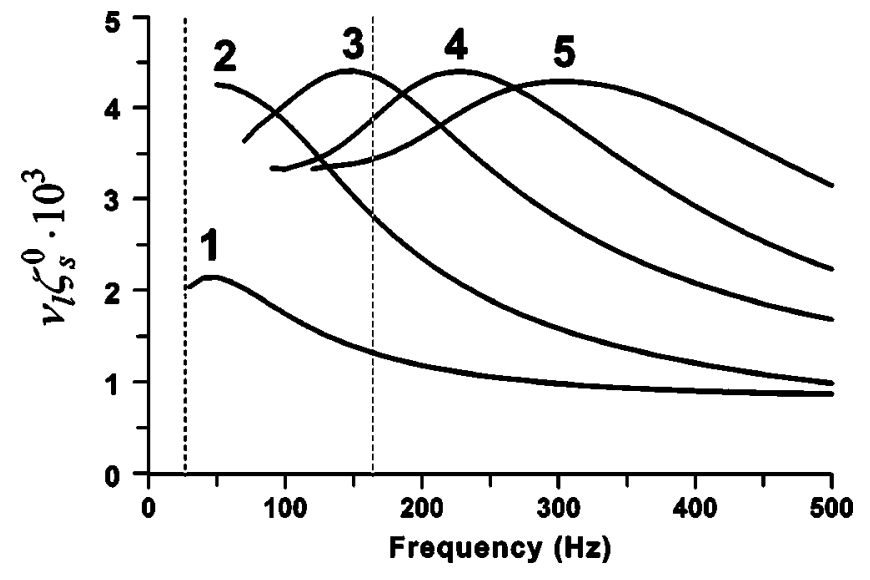

FIG. 8. Frequency-dependent index of refraction for horizontal rays at the maximum ISW amplitude $(10 \mathrm{~m})$, calculated for the five lowest vertical modes. Dashed lines indicate the approximate frequency band of interest in the airgun data $(30-160 \mathrm{~Hz})$.

An example is provided here of a simple estimate of the refraction index for an HR (as shown in Ref. 14), using numbers that are typical of SWARM. If $h_{t} / H \sim 0.2$, where $h_{t}$ is thickness of the thermocline, then the average Brunt-Vaisala frequency in that layer is $N_{0} \sim 12 \mathrm{cph}$ or $\sim 0.02 \mathrm{rad} / \mathrm{s}$. Consequently from Eq. (20), $\nu_{l} \sim 4 \times 10^{-4} \mathrm{~m}^{-1}$, so the correction to the squared refraction index in the horizontal plane is $\left|\bar{\mu}_{l}\right| \sim 4 \times 10^{-4} \zeta_{s}$. This value corresponds to the detailed calculations from Eq. (20) in Fig. 8.

The negative sign in Eq. (19) means that if a minimum displacement $(\zeta=0)$ falls on the source, then the refraction index has a local maximum at this point as a function of $y$. In this case HRs emitted from the source deviate toward the $x$ axis, denoted as "defocusing" [Fig. 9(a)]. If a maximum displacement (soliton peak) falls on the source, then "focusing" [Fig. 9(b)] occurs. Using the reciprocity principle, this argument also applies from the viewpoint of incoming rays. In the SWARM experiment, the available record of ISWs was obtained at the receiving array, so it is appropriate to apply these ideas for incoming rays.

To estimate how the space-time distribution of the sound field is affected by a passing ISW train using the HRs, consider an angular sector in the $x y$ plane where the emitted HRs (at angle $\chi_{0}$, as shown in Fig. 10) have turning points in the region between two peaks of the ISW train. This situation reflects the focusing, or increasing intensity within this region. Using the waveguide parameters appropriate for SWARM gives a critical angle estimate $\chi_{0} \sim 2 \sqrt{\left|\nu_{l} \zeta_{s}\right|}$ $\sim 8 \mathrm{deg}$ for this example. The maximum longitudinal scale $X_{0}$ for focusing is half a ray cycle distance in the horizontal plane for rays with deviation of order $\Lambda / 2$, where $\Lambda$ is the distance between adjacent peaks in the ISW train in the $y$ direction, as shown in Fig. 10. This can be estimated as

$$
X_{0}=\int_{0}^{-y} \frac{d y}{\operatorname{tg} \chi(y)}=\int_{0}^{\Lambda / 2} \frac{d y}{\sqrt{\nu_{l} \zeta_{s}(y)}} \sim \frac{2 \Lambda}{\chi_{0}} .
$$

For example, if $\Lambda \sim 400 \mathrm{~m}$ and $\zeta_{0} \sim 10 \mathrm{~m}$, then $X_{0} \sim 6 \mathrm{~km}$. This means that the "critical region" where the horizontal refraction can be manifested is within the sector with central angle $\chi_{0}$ and distances greater than $X_{0}$ from the source (see 
$\mathrm{Z}$ (m)

$10 \quad 0$

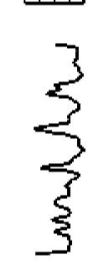

$z$ (m)

100

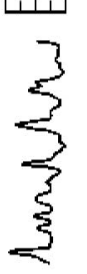

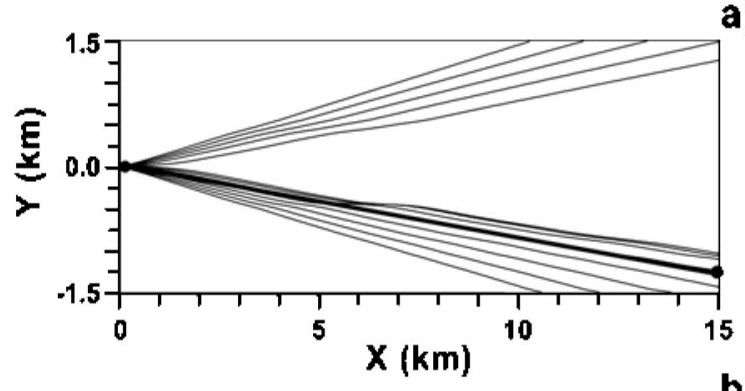

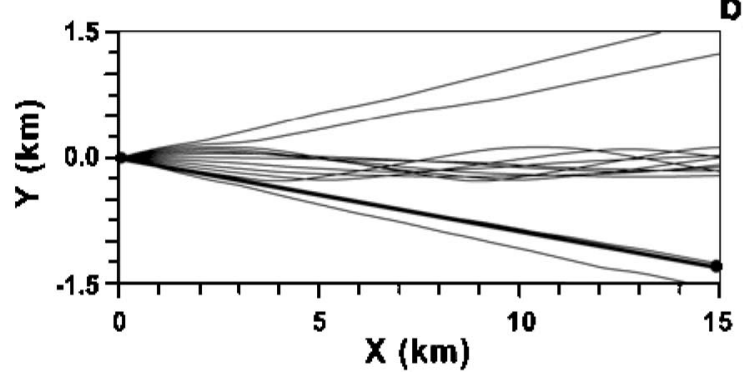

a)

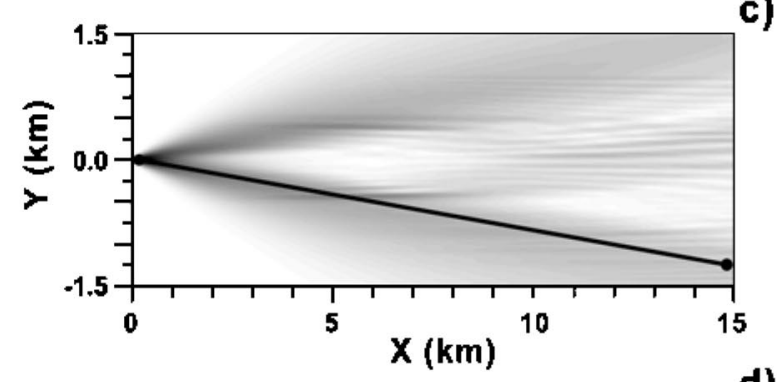

d)

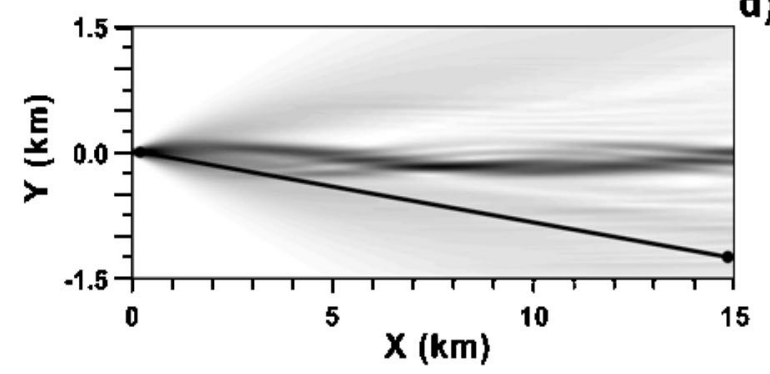

FIG. 9. (a) Ray theory calculation of the acoustic field in the horizontal plane corresponding to geotime 19:16 GMT, showing defocusing by horizontal refraction and an energy maximum; (b) same as (a), but corresponding to geotime 19:22 GMT, showing focusing and an energy minimum; (c) and (d), same as (a) and (b) but using the PE method. Corresponding ISW positions are shown to the left of each plot, and bold straight lines denote the source-receiver track.

Fig. 10). This explains why, in the SWARM experiment, the source-receiver separation and ISW parameters and positions (see Fig. 1) permit the influence of horizontal refraction on the sound field to be observed. In Fig. 9, HRs are presented for two geotimes (19:16 and 19:22 GMT) that correspond to a maximum and minimum in the time dependence of time-integrated intensity, as shown in Fig. 5. The intensity maximum corresponds to the case of defocusing, whereas the minimum (19:22 GMT) corresponds to focusing.

Using HRs one can roughly estimate the amplitude of

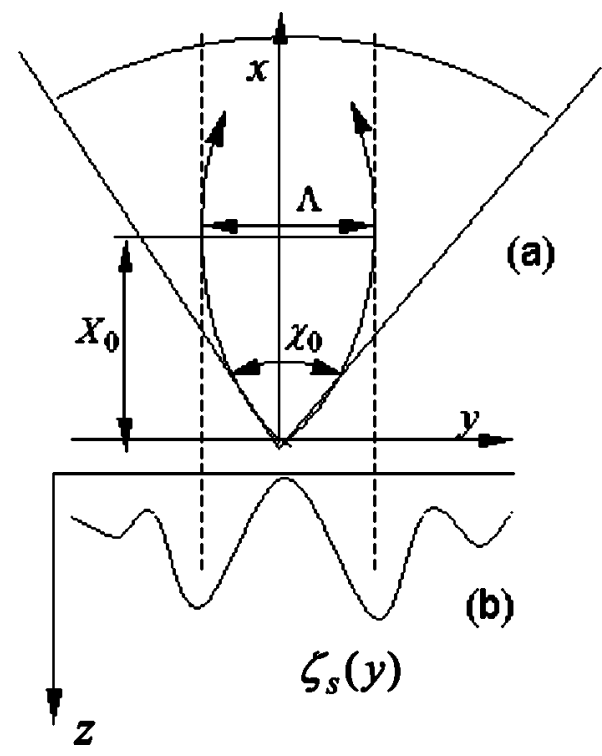

FIG. 10. Schematic diagrams showing: (a) patterns of horizontal rays with respect to the ISW amplitude, with dashed lines showing turning points of the rays focused within the critical angle $\chi_{0}$ and curved lines representing horizontal rays with launch angles near $\chi_{0}$; and (b) ISW amplitude in the $y z$ plane. the intensity fluctuations observed in Fig. 5 as a function of geotime for the experimental conditions. At a distance of 15 $\mathrm{km}$ from the source and using the estimated waveguide parameters calculated above, in the absence of ISWs a homogeneous intensity distribution would occur along the circular arc in Fig. 10. For the sector with central angle $\chi_{0} \sim 8 \mathrm{deg}$, the length of the arc is $\mathrm{L} \chi_{0} \sim 2 \mathrm{~km}$. For the focusing scenario the intensity in this area will concentrate between adjacent crests of the ISW. As noted above, this area has a length of $\sim 400 \mathrm{~m}$, so the intensity increases by approximately a factor of 5 , or $\sim 7 \mathrm{~dB}$. For the defocusing scenario the ratio of intensities tends to infinity. The experimentally observed fluctuation values may be somewhat smaller than the focusing estimate because the acoustic track direction is not exactly parallel to ISW crests, and the source and receiver are not located between the same peaks of the train. In summary, the proposed mechanism of sound fluctuations is the focusing/defocusing of HRs, which provides synchronicity over depth, correlation with ISWs, and time-integrated intensity fluctuation features that are observed in the data.

By considering the experiment from the viewpoint of reciprocity, one can construct incoming HRs toward the receiver in order to describe the propagation. Examples of such HRs are presented for defocusing [Fig. 11(a)] and focusing [Fig. 11(b)] cases. As described previously for case (b), the source falls close to the shadow zone of HRs coming toward receiver, and so an intensity minimum should occur. Correspondingly for case (a), the sound intensity should increase.

\section{B. Frequency dependence}

As discussed in the previous section, HR trajectories and amplitudes depend on frequency through the eigenvalues $q_{l}$ on the right side of Eq. (12). Therefore, in the integral of Eq. 

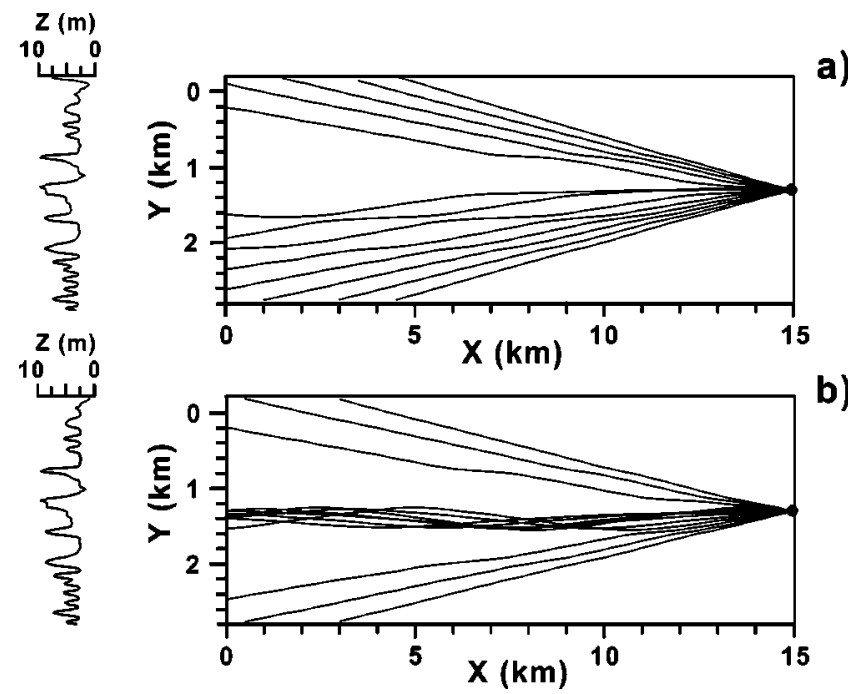

FIG. 11. Horizontal rays shown from the viewpoint of incoming rays at (a) 19:41, and (b) 19:47 GMT. Corresponding ISW positions are shown to the left of each plot. Note that the source position is at the origin for both cases.

(10), different contributions to the time-integrated intensity come from different frequencies. Recall that in Fig. 8 the magnitude of the refraction index is plotted versus frequency for five modes, with the vertical dashed lines representing the frequency band relevant to the SWARM airgun results.

To understand the propagation of broadband signals, the frequency dependence of the mode functions and of the HR, and their connections, need to be explained. From waveguide modal analysis, ${ }^{23}$ at a fixed frequency the higher-order modes have their maximum amplitudes closer to the sea surface and hence to the layer containing the ISWs. The maximum amplitudes of lower modes are well below this layer. Hence, the higher modes have a greater chance of overlapping the thermocline. ${ }^{15}$ As a function of increasing frequency, the maximum amplitude of each mode tends toward the sea bottom. Consequently, a frequency exists for which the amplitude of a particular mode overlaps with the internal wave layer. The existence of a maximum index of refraction at this frequency can be interpreted as having a number of HRs corresponding to a "quasiresonant" frequency, which increases their contributions to the sound field (in the case of focusing) and in turn provides higher intensity in comparison with other frequencies. Correspondingly in the case of defocusing, we should observe deeper minima for these quasiresonant frequencies. Away from this special frequency the effect of HRs reduces, as is shown clearly for modes 1 through 5 in Fig. 8. For some frequencies, an overlap occurs between the index of refraction of different modes. For example, in the band of interest in the SWARM experiment, (i.e., $30-160 \mathrm{~Hz}$ ) the refraction indices for modes 1 and 4 are below those of modes 2 and 3, while the latter overlap. This phenomenon causes frequency-selective behavior of the refraction index and is similar to chromatic aberration in optics, where the focusing properties of a lens depends on the frequency/color of light.

To apply this theoretical result for interpretation of experimental data requires caution, since the single WVLA shown in Fig. 1 limits the ability to extract spatial informa- tion. However, it is possible to confirm the frequency dependence using the temporal behavior of the spectral timeintegrated intensity. From the above reasoning the fluctuations due to the focusing/defocusing process increase with increasing refraction index. Thus, if separate frequency or modal components of the sound field are considered, these fluctuations will be greater for components having higher values of refraction index, which depends on mode number and frequency.

To illustrate this important point for SWARM, the spectrum of the received signals as a function of geotime for different modes is extracted from experimental data for the $30-160-\mathrm{Hz}$ band. The following processing of experimental data was carried out. After frequency filtering the received pulses $P(z, t ; T)$ with the sliding window $\Delta f=10 \mathrm{~Hz}$ for all geotime, a modal decomposition of the signal is performed

$$
P_{\omega}(z, t, T)=\sum_{l} \psi_{l}(z) a_{l \omega}(t, T) \cos \left(\omega t+\theta_{l}\right),
$$

where $P_{\omega}$ is a spectral component and $\theta_{l}$ is the phase shift. The modal time-integrated intensity for each frequency interval is calculated as

$$
I_{l \omega}(T)=\frac{1}{2} \int_{T}^{T+\Delta t} a_{l \omega}^{2}(t, T) d t .
$$

The results are shown in Fig. 12, which shows the fluctuation amplitude of each mode varying as a function of frequency, mode number, and geotime. For example, mode 1 has the largest fluctuations for the frequency band of $90-100 \mathrm{~Hz}$, while mode 3 shows the largest fluctuations for $150-160 \mathrm{~Hz}$. The character of the intensity fluctuation certainly differs for each mode as a function of frequency. Note that due to the frequency and mode number dependence of the refraction index, exact synchronicity of sound fluctuations for all modes and frequencies does not occur, as can be seen from Fig. 12. The synchronicity is revealed as a result of some averaging over frequency or space intervals.

To further interpret these results, we calculate the squared average value of the modal energy flux fluctuations after removing the mean. We use the concept of scintillation index (SI) defined for given mode number and frequency ${ }^{24}$ :

$$
S I_{l}^{2}(\omega)=\frac{\left\langle I_{l \omega}^{2}\right\rangle-\left\langle I_{l \omega}\right\rangle^{2}}{\left\langle I_{l \omega}\right\rangle^{2}}
$$

where $\langle F\rangle=1 / T_{0} \int_{0}^{T_{0}} F(T) d T$. This quantity characterizes the average fluctuations for the entire $T_{0}=1$-h period in Fig. 13, which shows results on a $\mathrm{dB}$ scale for the three lowest modes. By comparing these dependencies with the refraction index behavior in Fig. 8, the averaged value of fluctuations is seen to depend on frequency. For example, the amplitude of the first mode undergoes less fluctuations than the second mode for all frequency bands, which corresponds to Fig. 8 where the refraction index for the second mode is always greater than the first. The fluctuations of the third mode for frequencies less than $\sim 100 \mathrm{~Hz}$ are less than those of the second mode, while for frequencies greater than $\sim 100 \mathrm{~Hz}$ the opposite behavior occurs. This behavior is also similar to the frequency dependence of the refraction index in Fig. 8. 
$30-40$
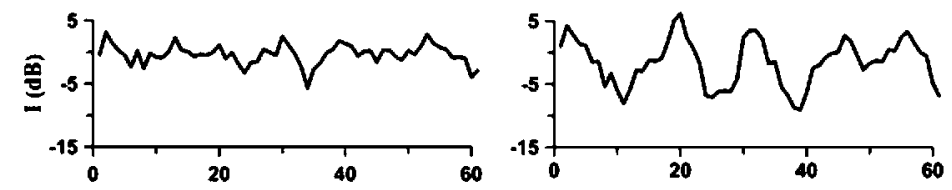

$60-70$
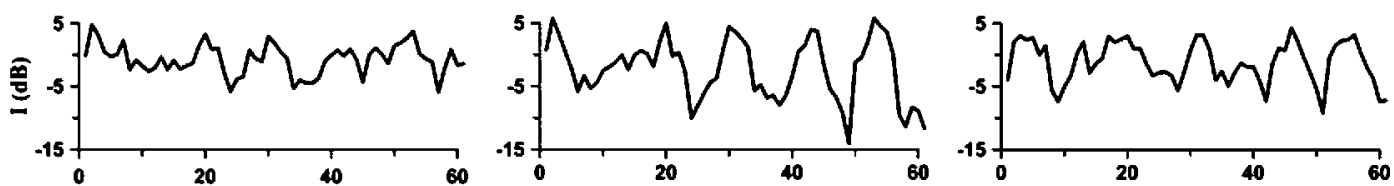

$90-100$
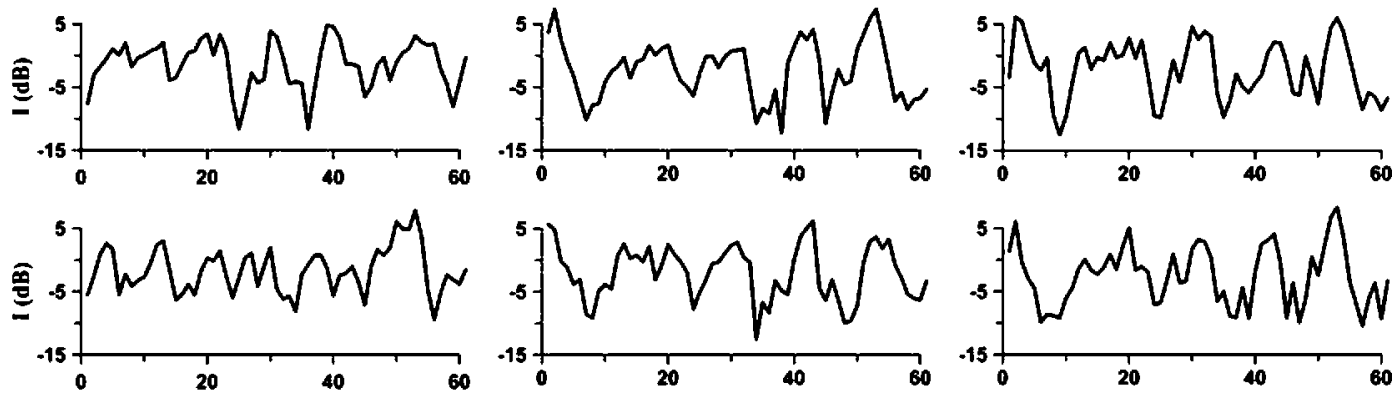

$150-160$
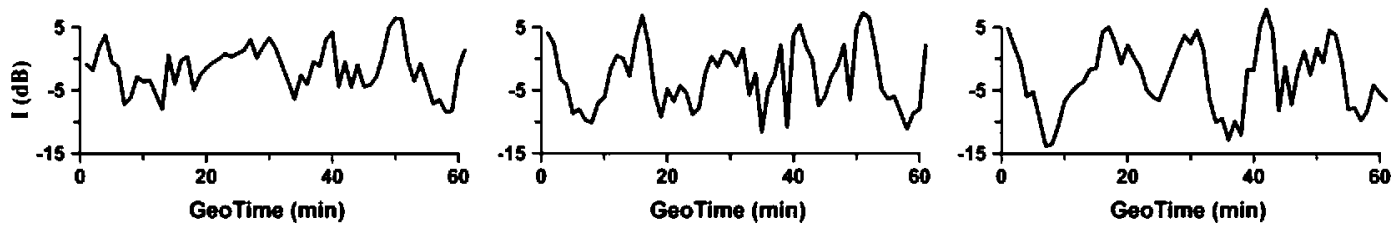

FIG. 12. Modal spectral time-integrated intensity $I_{i \omega}(T)$ in $\mathrm{dB}$ versus geotime for the three lowest modes and five spectral bands, calculated from data using Eq. (23).

Note the qualitative similarity between Figs. 8 and 13. Because of the frequency dependence, the curves in Fig. 13 will have the same shape at any receiver within the critical sector, because Eq. (20) depends on the unperturbed waveguide parameters only.

\section{Parabolic equation calculations in the horizontal plane}

The HR technique above provides a method to understand and interpret the basic features of sound propagation in the presence of horizontal refraction. However, HR calculations have the usual problems of ray caustics, so the theory is rarely applied for experimental comparisons. A more appro-

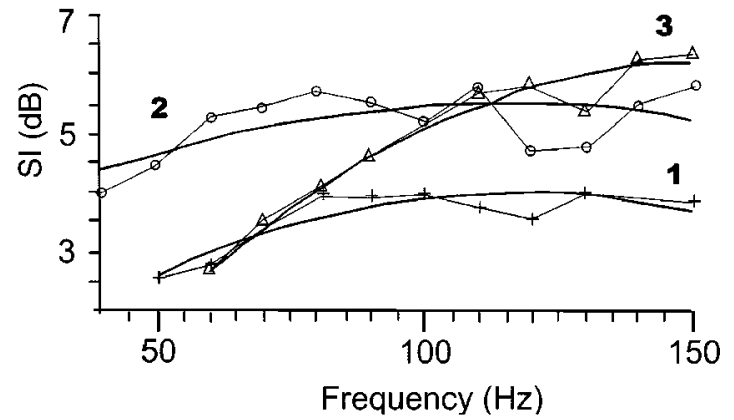

FIG. 13. Scintillation index, SI versus frequency for the three lowest modes; experimental values (points, joined by broken line) and their interpolation using mean square approximation (solid line). priate technique for this goal is the parabolic equation (PE) ${ }^{25}$ A version of this method known as the adiabatic mode PE (AMPE) was developed for 3D calculations without mode coupling. ${ }^{26}$

In order to compare calculations with the observed experimental fluctuations using this PE, the starting point is the received field for a fixed frequency as a function of geotime $T$ and source position $\Psi(\mathbf{r}, z, \omega)$. Equation (7) is rewritten with the new variable $P_{l}^{0}\left(\mathbf{r}_{r}, \mathbf{r}_{s}\right)$ introduced

$$
\Psi(\mathbf{r}, z, \omega)=\sum_{l} \psi_{l}\left(\mathbf{r}_{s}, z_{s}\right) \psi_{l}(\mathbf{r}, z) P_{l}^{0}\left(\mathbf{r}, \mathbf{r}_{s}\right) e^{-\left(\gamma_{l} / 2\right)\left|\mathbf{r}-\mathbf{r}_{s}\right|},
$$

where as before the source and receiver coordinates are $\left(\mathbf{r}_{s}, z_{s}\right)$ and $(\mathbf{r}, z)$, and $\gamma_{l}$ is the attenuation coefficient for mode $l$. This revision of Eq. (7), including the attenuation and source depth factors, is convenient for the PE solution. The solution of Eq. (9) is written in the form

$$
P_{l}^{0}\left(\mathbf{r}, \mathbf{r}_{s}\right)=F_{l}(x, y) \exp \left(i q_{l}^{0} x\right)
$$

where $F_{l}(x, y)$ is a slowly varying function in the propagation direction for each mode. Considering $F_{l}(x, y)$ in the forward-scattering approximation $\left(\partial F_{l} / \partial x \ll q_{l}^{0} F_{l}\right)$ leads to a $\mathrm{PE}$ in the horizontal plane

$$
\frac{\partial F_{l}}{\partial x}=\frac{i}{2 q_{l}^{0}} \frac{\partial^{2} F_{l}}{\partial y^{2}}+\frac{i q_{l}^{0}}{2} \mu_{l} F_{l} .
$$




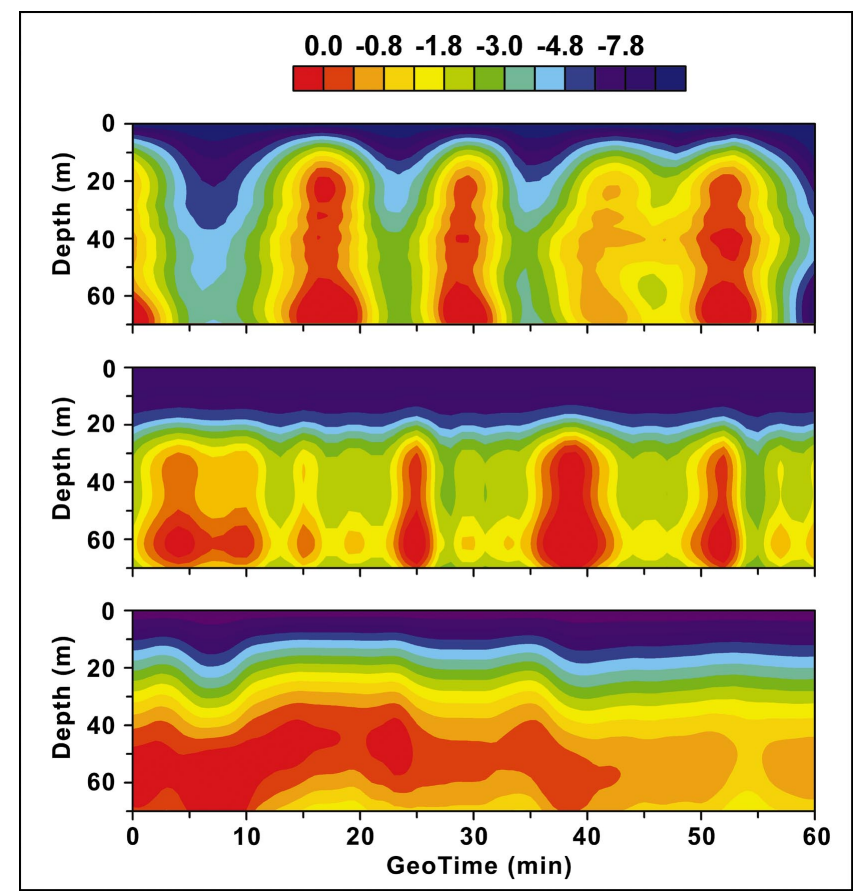

FIG. 14. Contour plots in depth and geotime for fluctuations of timeintegrated intensity in normalized units: (a) experimental results [top]; (b) modeling results using horizontal PE and vertical modes [middle]; (c) modeling results using adiabatic modes without horizontal refraction [bottom]. The color unit is in $\mathrm{dB}$.

The numerical solution of this equation can be produced with the standard split-step algorithm using the Fourier transform, although more accurate procedures ${ }^{26}$ use other methods.

As an example of calculating the modal amplitude in a horizontal plane for fixed frequency, the solution of Eq. (27) is presented for the same case as done with the HR method earlier. The results are presented in Figs. 9(c) and (d) for comparison with the HR calculations in Figs. 9(a) and (b). These plots show the distribution of the second vertical mode amplitude for frequency $100 \mathrm{~Hz}$. Excellent qualitative correspondence between results for the HR and PE methods is shown in depicting focusing and defocusing of the field, as is expected. To use this method for interpretation of and comparison with SWARM data, the spectral time-integrated intensity summed over all modes is calculated from the equation

$$
\begin{aligned}
I_{\omega}(\mathbf{r}, z)= & \frac{4 \pi}{\rho c}|S(\omega)|^{2} \sum_{l} \psi_{l}^{2}\left(\mathbf{r}_{s}, z_{s}\right) \psi_{l}^{2}(\mathbf{r}, z)\left|P_{l}^{0}\left(\mathbf{r}, \mathbf{r}_{s}\right)\right|^{2} \\
& \times e^{-\gamma_{l}\left|\mathbf{r}-\mathbf{r}_{s}\right|}
\end{aligned}
$$

For the geotime behavior of total time-integrated intensity, integrate Eq. (28) over frequency to obtain the analogue of Eq. (10)

$$
I(\mathbf{r}, z)=\int_{0}^{\infty} I_{\omega}(\mathbf{r}, z) d \omega .
$$

\section{COMPARISON OF DATA AND MODEL RESULTS}

A comparison between the experimental data and modeled results is shown in Fig. 14. Figure 14(a) shows contours of the total time-integrated intensity from Eq. (3) of the re- ceived acoustic signal on the WVLA from 19:00 to 20:00 GMT. This figure, which is constructed using all 16 hydrophones of the WVLA, clearly shows the 10- to 15-min periodicity of the intensity fluctuations versus geotime. In Fig. 14(b) the modeled results over the same geotime period are shown using calculations from the PE in Sec. IV-C. Five modes are used over the frequency band of 30-160 Hz, which is sufficient because nearly all the spectral energy is in the lowest part of this band and because higher-order modes contribute very little to the total acoustic field. Comparison of Figs. 14(a) and (b) confirms the 3D behavior of the waveguide in the presence of ISWs. The depth distribution of the time-integrated intensity, as well as the quasiperiodicity of the fluctuations, agrees well with the experimental data. Furthermore, very good agreement is shown for the depth locations of the intensity maxima, especially for the second half of the 1-h period. This is due to the passage of three large ISW peaks during this period. Differences between theoretical calculations and experimental data exist in the geotime arrivals of the maxima of the time-integrated intensity patterns (shifts of 3 to $5 \mathrm{~min}$ in arrival of the high-intensity bands near geotimes 19:25, 19:15, and 19:07 GMT in the calculated results). Other differences arise from the idealizations of the shallow-water waveguide model, such as the plane wavefront assumption for the ISW train. In the absence of detailed measurements of the ISW front curvature and other features, more precise matches with the experimental results are not expected. Moreover, the comparisons here between data and calculations are of one quantity, the total time-integrated intensity, which is expected to be more robust than others such as individual received pulses or their spectra.

The comparatively good agreement that is obtained for the positions of the last maxima in Fig. 14(b) can be discussed further. Within the framework of the plane wavefront assumption, three distinct small ISWs pass through the WVLA during the period 19:00 to 19:30 GMT, when large ISWs are detected at the source (see Fig. 9). From 19:30 to 20:00 GMT we have the opposite situation, with large ISWs at the receiver. The latter period can be modeled more realistically and naturally gives the predominant contributions to $3 \mathrm{D}$ effects. Moreover, the latter situation where the three maxima are visible at the receiver is well described by the incoming ray analogy (shown in Fig. 11). Some minor differences between the experimental data and the modeled results in Figs. 14(a) and (b) can be attributed to the lack of detailed environmental data along the acoustic track, including the bottom properties and the range-dependent soundspeed profile. However, it is noted that the modeling results presented here provide all the features of intensity fluctuations due to horizontal refraction.

Finally, Fig. 14(c) is presented to demonstrate conclusively the important role of horizontal refraction in the timeintegrated intensity fluctuations. These contours represent the results of model calculations for the same experimental period but without taking into account horizontal refraction. This is accomplished by using the $2 \mathrm{D}$ adiabatic mode approximation, ${ }^{21}$ which is valid because the sound-speed gradient along the acoustic track is relatively small, $\sim 6$ 
$\times 10^{-3} \mathrm{~s}^{-1}$. In this case the expression for $F_{l}(x, y)$ in Eq. (26) is found from Eq. (27) by neglecting the refraction term and using the appropriate source normalization. The corresponding formula for the spectral time-integrated intensity differs from Eq. (28) by replacing $\left|P_{l}^{0}\left(\mathbf{r}, \mathbf{r}_{s}\right)\right|^{2}$ with $1 /\left(8 \pi q_{l} L\right)$. The space-time intensity patterns in Fig. 14(c) are quite different from both the experimental results and the calculations including horizontal refraction. Figure 14(c) represents a $2 \mathrm{D}$ interference pattern with time-integrated intensity fluctuations of less than about $15 \%$. For example, in the temperature record of Fig. 2 near geotime $\sim 19: 30$ GMT, cold water occurs from a soliton displacement minimum. This thermocline rise broadens the effective sound channel and decreases intensity in the 2D calculations near $\sim 19: 34$. At $\sim 19: 36$ in Fig. 2 the water is warmer, the thermocline sinks corresponding to a soliton maximum, the sound channel narrows, and the sound intensity increases near $\sim 19: 41$. This is opposite to predictions from the $3 \mathrm{D}$ modeling and the experimental data.

\section{CONCLUSIONS}

When broadband acoustic signals propagate through ISWs, time-integrated intensity fluctuates with periods close to that of the ISW. Depending on the source-receiver geometry, the orientation of the internal wavefront with respect to the acoustic track, and other parameters of the waveguide such as the ratio between the thermocline layer thickness and the water depth, this effect could be significant (as large as $10 \mathrm{~dB}$ in the SWARM data presented here).

The experimental data in this paper indicate significant peak-to-peak total pulse-integrated intensity fluctuations of broadband signals. In addition, they are largely synchronous in water depth, so that the total signal energy in the water for a given geotime remains largely the same for all depths (below the thermocline). These features are interpreted as manifestations of 3D refraction in this shallow-water waveguide. To explain them, a theoretical model using horizontal rays and vertical modes is described. Results of the vertical mode and frequency spectrum decomposition of data are in strong agreement with model calculations. It is relevant to note that characteristics of the time-integrated intensity fluctuations can be qualitatively interpreted within the framework of ray theory. A direct comparison with integrated intensity data is facilitated using a horizontal plane PE model. The comparison between calculations and measured data shows good agreement, including the main features of the fluctuations. However, mismatches do occur, such as a time shift (up to $\sim 3$ min in Fig. 14) in the geotime dependence of integrated intensity. The differences result from idealizations in the waveguide model, which is based on data from thermistors at different depths along the receiver array. With only one point measurement, the ability to model properties, such as orientation, speed, and curvature, along the ISW wavefront is limited. It is noteworthy that, although small perturbations of the ISW speed and orientation angle modify the results, the synchronicity in depth and overall fluctuation amplitude is robust and does not vary significantly. Frequency-dependent focusing effects due to horizontal refraction are also found, with quite good agreement between theoretical and experimental results. This feature is analogous to chromatic aberration in optics.

Future work remains on this topic. Experimentally, a data set is needed that encompasses more frequencies, azimuthal angles, and ranges. Direct measurements of any ISW wavefronts would be extremely valuable. Theoretically, the structure of the horizontal ( $x-y$ plane) modes, both trapped and continuous, that is created between the ISWs should be investigated. The ray and PE representations, as well as rough estimates ${ }^{14}$ and numerical properties ${ }^{18}$ of this structure, have been carried out, but more detailed analysis of the horizontal modes should produce additional insights into the physics of this fascinating ducting effect.

\section{ACKNOWLEDGMENTS}

The authors wish to thank the participants of the SWARM'95 experiment for their efforts. This work was supported by the Ocean Acoustics Program at the Office of Naval Research (ONR Grants N00014-01-1-0114 to U.D., and N00014-04-1-0016 to R.P.I.) and by the Russian Foundation For Basic Research (RFBR Grant 03-05-64568-a). This manuscript is also a WHOI contribution.

\section{APPENDIX: Estimation of ISW speed V}

The ISW speed $V$ in the SWARM region can be estimated using some canonical models. For a water column with two layers, $H=h_{1}+h_{2}=20+50=70 \mathrm{~m}$, where each layer has constant temperature, select $\Delta T=T_{2}-T_{1}$ $\sim 13$ deg. It follows that $\Delta \rho \sim 1.7 \mathrm{~kg} / \mathrm{m}^{3}$, so the buoyancy frequency is $N \sim 10$ cycles/h. If the ISW train consists of separate KdV solitons, for each of which

$$
\zeta=\zeta_{0} \sec h^{2}[(y-V T) / \Delta]
$$

where $^{26}$

$$
V=c_{0}+\zeta_{0} \tilde{\alpha} / 3, \quad \Delta=\sqrt{12 \tilde{\beta} / \tilde{\alpha} \zeta_{0}},
$$

then

$$
\begin{aligned}
& c_{0}=\sqrt{g \frac{\Delta \rho}{\rho} \frac{h_{1} h_{2}}{H}}, \quad \tilde{\alpha}=c_{0} \frac{3\left(h_{1}-h_{2}\right)}{2 h_{1} h_{2}}, \\
& \tilde{\beta}=c_{0} \frac{h_{1} h_{2}}{6} .
\end{aligned}
$$

For SWARM conditions $c_{0} \sim 0.55 \mathrm{~m} / \mathrm{c}, \tilde{\beta} \sim 90 \mathrm{~m}^{3} / \mathrm{s}$, and $\tilde{\alpha}$ $\sim 0.024 \mathrm{~s}^{-1}$. Furthermore, if $\zeta_{0} \sim 10 \mathrm{~m}$, then $\Delta \sim 70 \mathrm{~m}$ and $V \sim 0.65 \mathrm{~m} / \mathrm{s}$. The differences between the speeds of separate solitons of different amplitudes in the train are ignored, so the whole train is assumed to move with the same speed.

\footnotetext{
${ }^{1}$ J. R. Apel, M. Badiey, C.-S. Chiu, S. Finette, R. H. Headrick, J. Kemp, J. F. Lynch, A. E. Newhall, M. H. Orr, B. H. Pasewark, D. Tielburger, A. Turgut, K. von der Heydt, and S. N. Wolf, "An overview of the SWARM 1995 shallow-water internal wave acoustic scattering experiment," IEEE J. Ocean. Eng. 22, 465-500 (1997).

${ }^{2}$ J. X. Zhou, X. S. Zhang, and P. H. Rogers, "Resonant interaction of sound waves with internal solitons in the coastal zone," J. Acoust. Soc. Am. 90, 2042-2054 (1991).

${ }^{3}$ D. Rubenstein and M. N. Brill, "Acoustic variability due to internal waves and surface waves in shallow water," in Ocean Variability and Acoustics
} 
Propagation, edited by J. Potter and A. Warn-Varnas (Kluwer Academic, Dordrecht, 1991), pp. 215-228.

${ }^{4}$ R. H. Headrick, J. F. Lynch, and the SWARM group, "Acoustic normal mode fluctuation statistics in the 1995 SWARM internal wave scattering experiment," J. Acoust. Soc. Am. 107, 201-220 (2000).

${ }^{5}$ R. H. Headrick, J. F. Lynch, and the SWARM group, "Modeling mode arrivals in the 1995 SWARM experiment acoustic transmissions," J. Acoust. Soc. Am. 107, 220-236 (2000).

${ }^{6}$ B. Pasewark, S. N. Wolf, M. H. Orr, and J. F. Lynch, "Acoustic intensity variability in a shallow water environment," in Impact of Littoral Environmental Variability on Acoustic Predictions and Sonar Performance, edited by N. Pace and F. Jensen (Kluwer Academic, Dordrecht, 2002), pp. $11-18$.

${ }^{7}$ M. Badiey, Y. Mu, J. F. Lynch, J. R. Apel, and S. N. Wolf, "Temporal and azimuthal dependence of sound propagation in shallow water with internal waves," IEEE J. Ocean. Eng. 27, 117-129 (2002).

${ }^{8}$ D. Rubenstein, "Observations of cnoidal internal waves and their effect on acoustic propagation in shallow water," IEEE J. Ocean. Eng. 24, 346357 (1999).

${ }^{9}$ J. C. Preisig and T. F. Duda, "Coupled acoustic mode propagation through continental shelf internal solitary waves," IEEE J. Ocean. Eng. 22, 256269 (1997).

${ }^{10}$ T. F. Duda and J. C. Preisig, "A modeling study of acoustic propagation though moving shallow-water solitary wave packets," IEEE J. Ocean. Eng. 24, 16-32 (1999).

${ }^{11}$ B. G. Katsnelson and S. A. Pereselkov, "Resonance effects in sound scattering by internal wave packets in a shallow sea," Acoust. Phys. 44, 684689 (1998).

${ }^{12}$ S. D. Frank, M. Badiey, J. F. Lynch, and W. L. Siegmann, "Analysis and modeling of broadband airgun data influenced by nonlinear internal waves," J. Acoust. Soc. Am. (to be published).

${ }^{13}$ Y. A. Kravtsov, V. M. Kuzkin, and V. G. Petnikov, "Perturbation calculation of the horizontal refraction of sound waves in a shallow sea," Sov. Phys. Acoust. 30, 45-47 (1984).

${ }^{14}$ B. G. Katsnelson and S. A. Pereselkov, "Low-frequency horizontal acous- tic refraction caused by internal wave solitons in a shallow sea," Acoust. Phys. 46, 774-788 (2000).

${ }^{15}$ B. G. Katsnelson, S. A. Pereselkov, V. G. Petnikov, K. D. Sabinin, and A. N. Serebryanyi, "Acoustic effects caused by high-intensity internal waves in a shelf zone," Acoust. Phys. 47, 424-429 (2001).

${ }^{16}$ B. G. Katsnelson and S. A. Pereselkov, "Space-frequency dependence of the horizontal structure of the sound field in the presence of intense internal waves,' Acoust. Phys. 50, 169-176 (2004).

${ }^{17}$ S. Oba and S. Finette, "Acoustic propagation through anisotropic internal wave fields: Transmission loss, cross-range coherence, and horizontal refraction," J. Acoust. Soc. Am. 111, 769-784 (2002).

${ }^{18}$ S. Finette and R. Oba, "Horizontal array beamforming in an azimuthally anisotropic internal wave field," J. Acoust. Soc. Am. 114, 131-144 (2003).

${ }^{19}$ D. Tielbuerger, S. Finette, and S. N. Wolf, “Acoustic propagation through an internal wave field in a shallow waveguide," J. Acoust. Soc. Am. 101, 789-808 (1997).

${ }^{20}$ S. M. Flatte, R. Dashen, W. M. Munk, K. M. Watson, and F. Zacharisen, Sound Transmission Through a Fluctuating Oceran, Cambridge Press, 1979.

${ }^{21}$ F. B. Jensen, W. A. Kuperman, M. B. Porter, and H. Schmidt, Computational Ocean Acoustics (Springer, New York, 2000).

${ }^{22} \mathrm{H}$. Weinberg and R. Burridge, "Horizontal ray theory for ocean acoustics," J. Acoust. Soc. Am. 55, 63-79 (1974).

${ }^{23}$ L. M. Brekhovskikh and Y. P. Lysanov, Fundamentals of Ocean Acoustics (Springer, New York, 1991).

${ }^{24}$ A. Ishimaru, Wave Propagation and Scattering in Random Media, Volume II, Academic Press, New York, 1978.

${ }^{25}$ F. D. Tappert, "The Parabolic Equation Method," in Wave Propagation and Underwater Acoustics, edited by J. B. Keller and J. S. Papadakis (Springer, New York, 1977), pp. 224-287.

${ }^{26}$ M. D. Collins, "The adiabatic mode parabolic equation," J. Acoust. Soc. Am. 94, 2269-2278 (1993).

${ }^{27}$ K. Naugolnykh and L. Ostrovsky, Nonlinear Wave Processes in Acoustics (Cambridge University Press, Cambridge, 1998). 